\title{
Telemedicine and Telehealth in the Era of COVID-19 and Beyond
}

\author{
Zainab J Gandhi ${ }^{1 *}$, Zabeen Mahuwala ${ }^{2}$ and Juzar $\mathrm{Ali}^{3}$ \\ ${ }^{1}$ Department of Medicine, C.U. Shah Medical College, India \\ ${ }^{2}$ Department of Neurology, University of Kentucky, United States \\ ${ }^{3}$ Section of Pulmonary/Critical Care/Allergy and Immunology, Department of \\ Medicine, Louisiana State University Health Sciences Center New Orleans, United \\ States \\ *Corresponding Author: Zainab J Gandhi, Department of Medicine, C.U. Shah \\ Medical College, India.
}

\author{
Received: September 29, 2020 \\ Published: November 10, 2020 \\ (C) All rights are reserved by Zainab J Gandhi., \\ et al.
}

\begin{abstract}
The COVID-19 pandemic has accelerated the implementation of telemedicine and its use for patient care. Telemedicine has been used in the past in many settings in non-crisis patient encounters and at many points of care, ranging from emergency room trauma units to clinic outpatient encounters in rural and semi-urban remote areas and as part of chronic care management. It has also been helpful in selected settings during natural disasters to improve access to care where conventional patient-doctor encounters were not possible. With its increased use during the current COVID-19 pandemic, it is important to review if this is truly a step forward in increasing access to care in challenging environments. This review article will attempt to outline the pros and cons of this emerging modality of healthcare delivery.
\end{abstract}

Keywords: Telemedicine; Telehealth; COVID-19; Clinical Examination

\section{Abbreviations}

COVID-19: Coronavirus Disease-19; ED: Emergency Department; ICU: Intensive Care Unit; OCT Machine: Optical Coherence Tomography; WBAN: Wireless Body Area Network; NSTEMI: Non- ST Elevation Myocardial Infarction; AIS: Alveolar Interstitial Syndrome; SARS-CoV2: Severe Acute Respiratory Syndrome- Related Coronavirus; PE: Physical Examination

\section{Background}

Telemedicine can be defined as the use of a remote electronic interface to provide connection with and care to the patients. It is a rapidly growing sector of healthcare delivery with recent market projections and network surveys that indicate telemedicine will account for $20 \%$ of medical visits, with physicians developing increased skills, and a financial impact of about $\$ 30$ billion. Telemedicine has hitherto primarily been practiced via provider to provider care in rural or non-specialist areas. However, the pa- tient-to-doctor connection is a rapidly evolving practice. There are essentially three types of telemedicine encounters: asynchronous, synchronous, and remote monitoring. Synchronous telemedicine involves patient care in real-time with live discussion and interaction between the patient and the provider. Asynchronous telemedicine involves a "store and forward technique," where the patient or the provider collects medical history, images, and pathology reports and this information is then sent to a specialist for expert advice. Remote patient monitoring refers to continuous monitoring of the patient's clinical status with the use of direct video monitoring or via reports received remotely through telemetry [1].

\section{Methodology}

Historically, the use of telemedicine and telehealth has been sparse and patchy throughout the United States. The use of telemedicine and its degree of synchronous interaction differs at each site of implementation across the globe. Physicians have been using 
informal phone connections to interact with patients for a long time and continue to rely on this communication for adequate history taking if such a need arises. However, with structured telemedicine and telehealth, there is the scope of improving physician-patient interaction and escalate it to the level of full physical examination in some fields. This nevertheless demands randomized control trials and more extensive studies to prove efficacy [2]. Handsch., et al. compared the traditional phone interview for stroke scale assessment with the remote audio-video examination and showed that without the use of video interface, there was more frequent hospitalization ( $9.1 \%$ vs. $14.9 \%, \mathrm{p}<0.05)$, a frequent misdiagnosis that had to be corrected ( $17.6 \%$ vs. $7.1 \%$; $<<0.05)$ and higher mortality when accessed after day 10 (6.8\% vs. 1.3\%, p < 0.05), thus proving the increased efficacy of having face-to-face gestures in providing finer medical engagement and outcome [3]. Use of telemedicine has been studied experimentally in various specialties of medicine [4]. Another prospective study was done to evaluate the use of telemedicine with real-time interpretation by out-of-hospital cardiologists with on-site focused echocardiography by nurses. Results showed that this telemedicine approach was feasible in $94 \%$ of the cases in assessment and quantification of the left atrium, left ventricle volume, dimensions, and functional indices [5]. For specialized testing such as ophthalmological examination, devices like slit-lamps, fundus cameras (non-mydriatic) and other examination modalities can be remotely controlled with some exceptions. Further, tele-visits could also help triage the patient's diagnosis and gauge the need for an in-person visit [6]. In the ER and urgent care where there may be an unknown and or maximum exposure to infectious agents, telemedicine can be useful to screen patients before their visit and minimize exposure to the health care staff. Telehealth monitoring in ICU settings also showed better outcomes in patients who were delayed in being transferred to advanced care in comparison with patients not being monitored via electronic ICUs [7].

Telehealth has been applied in many alternative ways. An advance application of telehealth would be Wireless body area network (WBAN) which is remote monitoring tool with autonomous sensor technology. In the ICU's, it could help in monitoring the patients in real-time $[8,9]$. Another method of use of telemedicine could include ICU Robots. These have been tested to be used in trauma ICUs to study the adaptability, teamwork, and behaviors of staff for patient rounds [10].

\section{Discussion}

Conventionally, face-to-face medicine has always involved a patient-centered physical examination and a closer clinical in-person interaction. It is individualistic and helps develop a physicianpatient relationship while aiding in diagnosis. Telemedicine offers similar experience but lacks the benefit of a comprehensive physical exam in most medical fields unless it can be accompanied by technologically easy to use tools that will aid in the physical exam. The role of physical examination (PE) goes beyond supplementing diagnostic accuracy [11]. It is also a benchmark in the prognosis of many diseases such as detecting stroke severity with a functional neurologic defect or in cardiology practice for NSTEMI with Killip classification despite inter-observer variation [12]. Studies have shown no difference in face-to-face encounters and tele-visit inpatient experience in certain specialties such as dermatology $[13,14]$.

In the past, telemedicine has been used in the SARS epidemic [15]. With the COVID-19 pandemic, the utilization of telehealth spiked from $11 \%$ to $46 \%$ to overcome the canceled visitation [16]. A survey showed $20 \%$ of ED visitations can be avoided with telemedicine or virtual urgent care offerings, and $24 \%$ of healthcare office visits and outpatient volume could be delivered virtually plus $9 \%$ "almost virtually". Also, up to $35 \%$ of regular home health attendant services could be virtualized, and $2 \%$ of all outpatient volume could be shifted to the home setting, with technology-based medication administration [17]. A single-center study in an urban hospital ED proved that the protocol to screen stable patients via telemedicine could be useful, especially during the COVID-19 pandemic [18]. In the Accenture 2019 Health Consumer Survey, consumers are leaning towards more remote management, especially in the Millennial and Generation $\mathrm{Z}$ consumers for convenience and affordability with transparency. Consumer surveys showed the same level of satisfaction for an in-person visit and remote communication, provided it is associated with digital capabilities [19]. With telemedicine, virtual consults can also be conducted with sharing the screen with the patients. A prospective study showed about $85 \%$ of the consultants of various specialties agreed virtual consultations were easy to use, saved time and improved patient management especially in cases of barriers such as physical distance or patient disability and in management of chronic diseases such as diabetes and arthritis [20]. Telemedicine can also be helpful in medication reconciliation which remains a complicated process at the time of patient discharge to gauge drug interactions and 
potential medication abuse. Preliminary studies done with the MedRec app showed promising results to explore the possibility [21].

Considering the COVID-19 pandemic, the federal government has taken initiatives to relax the restrictions on telehealth. Various factors, such as higher internet speed (5G data), electronic medical records, and various platforms of communication has thus helped the rapid expansion of telemedicine in recent months. Telehealth and Medicare partnered to provide secure billing for reimbursement of tele visits with billing codes to ease the process [22].

\section{Challenges going forward}

Telemedicine does come with its own set of challenges. Adaptation to telemedicine needs additional training, knowledge of the complexity of various technical and troubleshooting processes, the capability to switch to virtual platform/s and above all clinical acceptance. Continuous funding for telehealth also remains a challenge post-pandemic, and the federal government will have a role to play in broad-scale implementation through centralized funding and regulations for proper reimbursement for the providers. Lastly, implementation of telehealth requires systematic strategies as telemedicine becomes integral to patient care with technological infrastructure especially in the case of robots, networking system along with standardized operations protocols [23]. With the rapid incorporation of telemedicine and telehealth in the delivery of patient care, there needs to be a focus on streamlining and standardizing the methods of care and setting up healthcare delivery systems for future sustainability. COVID -19 pandemic has at times put a tremendous burden on the surge capacity of the health delivery system and increased the challenges of screening patients and personnel in highly infectious zones. This is specially important when availability of appropriate personal protective equipment maybe limited [24]. Telemedicine not only was brought to use in these settings but also enabled the continuity of non-COVID 19 related patient care to some extent [25]. There is thus a global call and need for implementation of a robust telemedicine infrastructure that encompasses all elements of clinical face to face interaction. It also remains essential to standardize the integration, regulation, communication, data sharing, and evaluation framework for telemedicine on a system level to address the existing pitfalls [26].

\section{Conclusion}

Various efforts have been put forth so far to implement telemedicine. While in the past, guidelines have been relaxed to incorporate telemedicine in crisis situations, it is imperative to assess the efficacy and practicality of using tele medicine in daily medical prac- tice on a long term-sustained basis. Studies and surveys done so far support the implementation of tele medicine, but logistic and other barriers need to be identified and removed before tele medicine and tele health can be used in a manner that matches the synergy of a face-to face acute and chronic care encounters in all medical specialties. It is only then will this modality achieve the full complement of care for the patient and provider.

\section{Conflict of Interest}

None.

\section{Bibliography}

1. Oren J Mechanic and Alexa B Kimball. "Telehealth systems". Stapearls Internet (2020).

2. Greenhalgh T., et al. "Virtual online consultations: advantages and limitations (VOCAL) study". BMJ Open 6.1 (2016): e009388.

3. Handschu R., et al. "Telemedicine in acute stroke: remote video-examination compared to simple telephone consultation". Journal of Neurology 255.11 (2008): 1792-1797.

4. Elphick HE., et al. "Validity and reliability of acoustic analysis of respiratory sounds in infants". Archives of Disease in Childhood 89.11 (2004): 1059-1063.

5. Hjorth-Hansen AK., et al. "Feasibility and Accuracy of TeleEchocardiography, With Examinations by Nurses and Interpretation by an Expert via Telemedicine, in an Outpatient Heart Failure Clinic" (2020).

6. Saleem SM., et al. "Virtual Ophthalmology: Telemedicine in a Covid-19 Era". American Journal of Ophthalmology (2020): 237-242.

7. Khairat S., et al. "Interpreting COVID-19 and Virtual Care Trends: Cohort Study". JMIR Public Health Surveill 6.2 (2020): e18811.

8. Gambhir S and Kathuria M. "DWBAN: Dynamic priority based WBAN architecture for healthcare system". 2016 3rd International Conference on Computing for Sustainable Global Development (INDIACom) (2016): 3380-3386.

9. Yuan X., et al. "On energy saving in e-healthcare: a directional MAC protocol for WBAN”. In: 2015 IEEE Globecom Workshops, GC Wkshps (2015).

10. Becevic M., et al. "Robotic Telepresence in a Medical Intensive Care Unit--Clinicians' Perceptions”. Perspectives in Health Information Management 12 (2015): 1c. 
11. Zaman JAB. "The Enduring Value of the Physical Examination". Medical Clinics of North America 102.3 (2018): 417-423.

12. Hansen M., et al. "Interobserver variation in the evaluation of neurological signs: observer dependent factors". Acta Neurologica Scandinavica 90.3 (1994): 145-149.

13. Holtz BE. "Patients Perceptions of Telemedicine Visits Before and After the Coronavirus Disease 2019 Pandemic". Telemedicine Journal and e-Health (2020).

14. Finnane A., et al. "Teledermatology for the Diagnosis and Management of Skin Cancer: A Systematic Review". JAMA Dermatology 153.3 (2017): 319-327.

15. Smith AC., et al. "Telehealth for global emergencies: Implications for coronavirus disease 2019 (COVID-19)". Journal of Telemedicine and Telecare (2020).

16. McKinsey COVID-19 Consumer Survey (2020).

17. Telehealth: A quarter billion-dollar reality? (2020).

18. Chou E., et al. "Onsite telemedicine strategy for coronavirus (COVID-19) screening to limit exposure in ED". Emergency Medicine Journal (2020).

19. Accenture Digital Health Consumer Survey (2020).

20. Rosenkrantz AB., et al. "Technology-Assisted Virtual Consultation for Medical Imaging". Journal of the American College of Radiology 13.8 (2016): 995-1002.

21. Center of Connected Health Policy (2020).

22. Marien S., et al. "A User-Centered design and usability testing of a web-based medication reconciliation application integrated in an eHealth network". International Journal of Medical Informatics 126 (2019): 138-146.

23. Smith AC., et al. "Telehealth for global emergencies: Implications for coronavirus disease 2019 (COVID-19)". Journal of Telemedicine and Telecare (2020): 1357633X20916567.

24. Tirupathi R., et al. "Comprehensive review of mask utility and challenges during the COVID-19 pandemic". Infez Medicine 28.1 (2020): 57-63.

25. Chou E., et al. "Onsite telemedicine strategy for coronavirus (COVID-19) screening to limit exposure in ED". Emergency Medicine Journal (2020).
26. Ohannessian R., et al. "Global Telemedicine Implementation and Integration Within Health Systems to Fight the COVID-19 Pandemic: A Call to Action". JMIR Public Health and Surveillance 6.2 (2020): e18810.

\section{Assets from publication with us}

- Prompt Acknowledgement after receiving the article

- Thorough Double blinded peer review

- Rapid Publication

- Issue of Publication Certificate

- High visibility of your Published work

Website: www.actascientific.com/

Submit Article: www.actascientific.com/submission.php

Email us: editor@actascientific.com

Contact us: +919182824667 